\title{
Metformin and intravenous contrast
}

\section{Mark Otto Baerlocher MD, Murray Asch MD, Andy Myers MDCM}

\section{Metformin is excreted by the kidneys}

Metformin is used in type 2 diabetes mellitus to decrease the amount of glucose produced by the liver and to increase the body's response to insulin. In patients with renal failure (acute or chronic), the renal clearance of metformin is decreased, and there is an associated risk of lactic acidosis, which has a mortality rate of up to $50 \%$. $^{1}$ Some patients who receive intravenous contrast may experience a deterioration of renal function (contrast-induced nephropathy). Although the points in this article discuss the use of intravenous contrast, the same principles apply to intra-arterial contrast.

For most patients, metformin should be stopped at the time of contrast administration

There is some controversy about when to stop and restart metformin for patients scheduled to undergo intravenous contrast-enhanced examinations. ${ }^{4}$ The guidelines from the Canadian Association of Radiologists ${ }^{2}$ state that patients taking metformin who have an estimated glomerular filtration rate (eGFR) of less than $60 \mathrm{~mL} / \mathrm{min}$ should stop taking metformin at the time of contrast administration. The European Society of Urogenital Radiology advocates stopping metformin 48 hours before CT for patients with an eGFR of less than $45 \mathrm{~mL} / \mathrm{min}^{5}{ }^{5}$

\section{Restarting metformin depends on renal function and the volume of contrast used}

Guidelines from the Canadian Association of Radiologists ${ }^{2}$ state that patients taking metformin who have an eGFR of less than $60 \mathrm{~mL} / \mathrm{min}$ should restart the drug no sooner than 48 hours after contrast administration and only if renal function remains stable $(<25 \%$ increase in creatinine above baseline). Patients with an eGFR above $60 \mathrm{~mL} / \mathrm{min}$ who receive a larger amount of intravenous contrast (> $100 \mathrm{~mL}$; e.g., CT of the abdomen or pelvis, CT angiography of the aorta or lower extremities) should restart metformin no earlier than 48 hours after the procedure. ${ }^{3}$

\section{Use of metformin is not a contraindi- cation to intravenous contrast administration}

Metformin in isolation is not considered a risk factor for contrast-induced nephropathy, ${ }^{2}$ but particular attention must be paid to patients taking metformin who are scheduled to undergo contrast-enhanced examination (e.g., enhanced computed tomography [CT], angiography, venography). ${ }^{3}$ Many physicians are particularly cautious in the case of elderly patients aged greater than 80 years.

For small volumes of contrast, patients with normal renal function taking metformin may not require any changes in care

If patients with normal renal function who are taking metformin receive less than $100 \mathrm{~mL}$ of intravenous contrast (e.g., enhanced CT of the brain), stopping metformin and/or rechecking creatinine levels 48 hours after the procedure may be unnecessary, because the risk of contrast-induced nephropathy in patients with normal renal function is very low. ${ }^{5}$

\section{References}

1. Misbin RI, Green L, Stadel BV, et al. Lactic acidosis in patients with diabetes treated with metformin. N Engl J Med 1998;338:265-6.

2. Benko A, Fraser-Hill M, Magner P, et al.; Canadian Association of Radiologists. Canadian Association of Radiologists: consensus guidelines for the prevention of contrast-induced nephropathy. Can Assoc Radiol J 2007;58:79-87.

3. Thomsen HS, Morcos SK. Contrast media and metformin. Guidelines to diminish the risk of lactic acidosis in non-insulin dependent diabetics after administration of contrast media. Eur Radiol 1999;9:738-40.
4. Goergen SK, Rumbold G, Compton G, et al. Systematic review of current guidelines, and their evidence base, on risk of lactic acidosis after administration of contrast medium for patients receiving metformin. Radiology 2010; 254:261-9.

5. Stacul F, van der Molen AJ, Reimer P, et al.; Contrast Media Safety Committee of European Society of Urogenital Radiology (ESUR). Contrast induced nephropathy: updated ESUR Contrast Media Safety Committee guidelines [review]. Eur Radiol 2011;21:2527-41.
Competing interests: None declared.

This article has been peer reviewed.

Affiliations: From the Department of Radiology (Baerlocher), Royal Victoria Hospital, Barrie, Ont.; and the Department of Radiology (Asch, Myers), Lakeridge Health Corporation, Oshawa, Ont.

Correspondence to: Mark Otto Baerlocher, mark.baerlocher@alumni.utoronto.ca

CMAJ 2013 DOI:10.1503/cmaj.090550 